\title{
Giant enhancement of cathodoluminescence of monolayer transitional metal dichalcogenides semiconductors
}

Shoujun Zheng, ${ }^{\dagger}, \#$ Jin-Kyu So, ${ }^{\dagger}, \#$ Fucai Liu,${ }^{\ddagger}$ Zheng Liu, ${ }^{\ddagger}$ Nikolay Zheludev $, *, \dagger, \S$ and Hong Jin Fan ${ }^{*}, \dagger, \|$

${ }^{\dagger}$ Centre for Disruptive Photonic Technologies, School of Physical and Mathematical Sciences \& The Photonics Institute, Nanyang Technological University, Singapore 637371

${ }^{\ddagger}$ School of Materials Science and Engineering, Nanyang Technological University, 639798, Singapore

${ }^{\S}$ Optoelectronics Research Centre and Centre for Photonic Metamaterials, University of Southampton, Southampton SO17 1BJ, United Kingdom

"Division of Physics and Applied Physics, School of Physical and Mathematical Sciences, Nanyang Technological University, 637371, Singapore 


\begin{abstract}
:
Monolayer two-dimensional transitional metal dichalcogenides, such as MoS2, WS and WSe2, are direct band gap semiconductors with large exciton binding energy. They attract growing attentions for opto-electronic applications including solar cells, photo-detectors, light-emitting diodes and photo-transistors, capacitive energy storage, photodynamic cancer therapy and sensing on flexible platforms. While light-induced luminescence has been widely studied, luminescence induced by injection of free electrons could promise another important applications of these new materials. However, cathodoluminescence is inefficient due to the low cross-section of the electron-hole creating process in the monolayers. Here for the first time we show that cathodoluminescence of monolayer chalcogenide semiconductors can be evidently observed in a van der Waals heterostructure when the monolayer semiconductor is sandwiched between layers of hexagonal boron nitride (hBN) with higher energy gap. The emission intensity shows a strong dependence on the thicknesses of surrounding layers and the enhancement factor is more than $\mathbf{5 0 0}$ folds. Strain-induced exciton peak shift in the suspended heterostructure is also investigated by the cathodoluminescence spectroscopy. Our results demonstrate that $\mathrm{MoS}_{2}, \mathrm{WS}_{2}$ and $\mathrm{WSe}_{2}$ could be promising cathodoluminescent materials for applications in single-photon emitters, high-energy particle detectors, transmission electron microscope displays, surface-conduction electron-emitter and field emission display technologies.
\end{abstract}

Keywords: 2D materials, van der Waals heterostructures, cathodoluminescence, carrier confinement, photoluminescence 


\section{Introduction}

Two-dimensional (2D) layered semiconductors, due to their weak interlayer van der Waals bonds, can be easily thinned down to atomic thickness by mechanical ${ }^{1}$ and chemical $^{2}$ exfoliation methods, for example, graphene ${ }^{3}$ and hexagonal boron nitride $(\mathrm{hBN})^{4}$. Monolayer transitional metal dichalcogenides (TMDCs) with the formula of $\mathrm{MX}_{2}(\mathrm{M}=\mathrm{Mo}, \mathrm{W} ; \mathrm{X}=\mathrm{S}, \mathrm{Se}$, Te) are a type of unique semiconductors with narrow direct band gaps, large exciton binding energies, high optoconductivity, and high photoelectrochemical activity. Moreover, due to the inversion symmetry breaking, monolayer $\mathrm{MX}_{2}$ are widely employed for the study of valley polarization and spin-valley coupling ${ }^{5,6}$. Recently, van der Waals heterostructures, composed of different 2D materials with unique band alignment and interlayer coupling, attract growing attentions not only for fundamental new physics but also for many potential applications such as tunneling transistors ${ }^{7-9}$ and light-emitting diodes ${ }^{10}$.

Considering the low dimensionality and unavoidable defects, grain boundaries, and local strain of 2D TMDCs, it is essential to characterize their electronic, structural, and optical properties with the spatial resolution down to the relevant length scales which are mostly at the nanoscale. With the well-established scanning tunneling microscopes ${ }^{11}$ or transmission electron microscopes ${ }^{12}$, characterizing the electronic and structural properties of monolayer TMDCs has been straightforward down to the atomic-scale. However, the optical characterization of excitonic excitations in monolayer TMDCs by photoluminescence (PL) is diffraction-limited due to the spot size of a laser, which should be resolved by creating optical excitations with sub-wavelength probes such as scanning probes ${ }^{13}$ or focused electron beams.

Cathodoluminescence (CL), photon emission excited by a high-energy electron beam, is widely applied in the analysis of mineral compositions ${ }^{14}$, light emitting diodes ${ }^{15}, 16$, surface plasmon mapping ${ }^{17}$. Compared to PL excited by light, CL offers a much higher excitation energy allowing the study of wide band gap materials including diamond ${ }^{18}$ and hexagonal boron nitride ${ }^{19,20}$. Due to a small excitation hotspot CL has been extensively used to study nanostructures including hyper-spectral imaging of plasmonic gratings ${ }^{21}$, nanoparticles ${ }^{22}$, nano-antenna ${ }^{23}$, quantum well ${ }^{24,25}$, three-dimensional nanoscale visualization of metaldielectric nanoresonators ${ }^{26}$ and nanoscale light sources ${ }^{27,28}$. Therefore, CL is an ideal candidate to investigate optical properties of 2D TMDCs in nanoscale. 
In monolayers of TMDCs, it is challenging to detect the interband CL signal as the electronhole creation cross section is extremely small. Moreover, the spatial distribution of electronhole pairs at the interface, which is near the point of free-electron injection, is close to a threedimensional spherical shape of a few microns in diameter. Only a negligible fraction of recombination takes place in the top 2D material and most of them happen in the supporting slab. So far only a few reports are available on CL study of 2D materials, including six atomic layer thick flakes of boron nitride. ${ }^{29-31}$ However, observation of CL from monolayer TMDCs has not been achieved.

In this report we show that CL emissions from monolayer $\mathrm{MX}_{2}\left(\mathrm{MoS}_{2}, \mathrm{WS}_{2}\right.$ and $\left.\mathrm{WSe}_{2}\right)$ can be enhanced and efficiently detected in a van der Waals heterostructure, in which the luminescent $\mathrm{MX}_{2}$ layer is sandwiched between layers of hexagonal boron nitride (hBN) with higher energy gap (see schematics in Figure 1a). Here the $\mathrm{hBN} / \mathrm{MX}_{2} / \mathrm{hBN}$ heterostructure can effectively increase the recombination probability of electron-hole pairs in the monolayer $\mathrm{MX}_{2}$ in such a way that a good fraction of the electrons and holes generated in the $\mathrm{hBN}$ layers diffuse to and then radiatively recombine in the $\mathrm{MX}_{2}$ layer, leading to significant enhancement of the emission, comparatively to an isolated layer (Figure 1b).
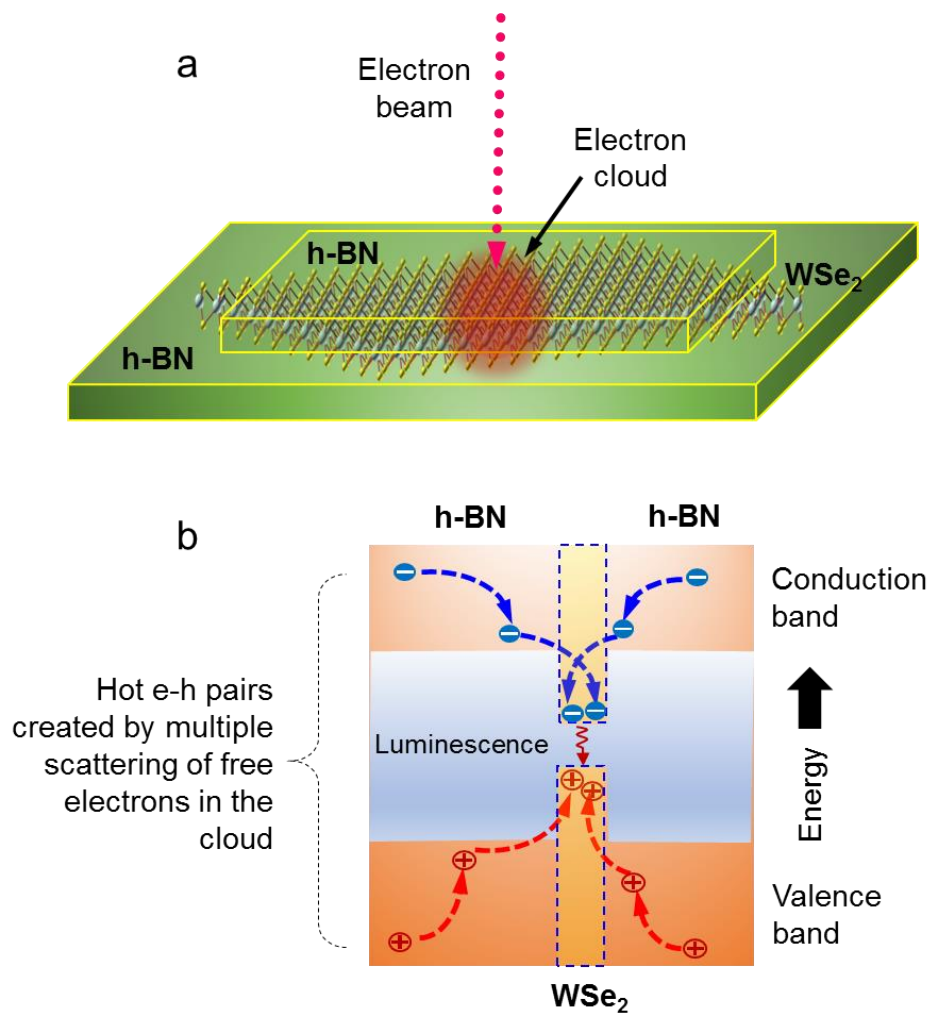

Figure 1. Electron beam induced light emission in a TMDC heterostructure. (a) Illustration 
of cathodoluminescence in an $\mathrm{hBN} / \mathrm{MX}_{2} / \mathrm{hBN}$ van der Waals heterostructure. (b) Process of the generation, diffusion and recombination of electron-hole (e-h) pairs. The minor number of e-h pairs generated in the $\mathrm{MX}_{2}$ layer is ignored.

\section{Cathodoluminescence of $\mathrm{hBN} / \mathrm{monolayer} \mathrm{WSe} 2 / \mathrm{hBN}$ heterostructure}

Heterostructures of $\mathrm{hBN} / \mathrm{TMDC} / \mathrm{hBN}$ were prepared by stacking individual flakes with a dry transfer technique ${ }^{1,32}$, where mechanically exfoliated flakes are picked up in sequence by van der Waals forces (see 'Methods' and Supporting Information Figure S1). Molybdenum disulphide $\left(\mathrm{MoS}_{2}\right)$, tungsten disulphide $\left(\mathrm{WS}_{2}\right)$ and tungsten diselenide $\left(\mathrm{WSe}_{2}\right)$ were tested as TMDC monolayers. Figure $2 \mathrm{a}$ is an optical image of a heterostructure $\mathrm{hBN} / \mathrm{WSe} / \mathrm{hBN}$ at an intermediate stage, where $\mathrm{hBN}\left(4.2 \mathrm{~nm}\right.$ thick) and $\mathrm{WSe}_{2}$ layers are clearly identified with an overlapped region. The heterostructure is completed by stamping this stack on another hBN layer ( $100 \mathrm{~nm}$ thick) on a $\mathrm{SiO}_{2} / \mathrm{Si}$ substrate as in Figure $2 \mathrm{~b}$. The TMDC monolayer and hBN layers constituting the heterostructures were further identified with micro-Raman (Supporting Information Figure S2).

Secondary electron imaging and CL measurements of prepared samples were performed in a scanning electron microscope equipped with a dedicated CL detection system. The emitted light induced by electron beam irradiation onto samples was collected by an achromatic reflective objective with a high numerical aperture (NA 0.72) and sent to a UV-VIS spectrometer equipped with a thermoelectrically cooled silicon CCD array. To produce hyperspectral images ${ }^{21}$, the focused electron beam (electron energy $5 \mathrm{keV}$; beam current $\sim 36$ $\mathrm{nA}$; dwell time 10-50 ms) scanned the samples according to a pre-defined resolution of the image while recording the light emission spectrum synchronously.

Figure 2c shows the CL intensity map of the heterostructure hBN/WSe $2 / \mathrm{hBN}$ near 1.572 $\mathrm{eV}$ which corresponds to the exciton energy of monolayer $\mathrm{WSe}_{2}$. Here, $\mathrm{WSe}_{2}$ monolayer glows only if sandwiched by the two hBN layers, which confirms the model for enhancing CL in monolayer TMDCs described in Figure 1. The extra e-h pairs created in the adjacent hBN layers diffuse into, trapped and recombine in the monolayer TMDCs. The CL spectra from $\mathrm{WSe}_{2}$ monolayer on (position 1) and off (position 2) the heterostructure is shown in Figure 2d. The small redshift of CL spectrum, $16.8 \mathrm{meV}$, from the PL spectrum in Figure $2 \mathrm{~d}$ is attributed 
to the temperature-induced band gap shrinkage ${ }^{33}$ of the semiconductor caused by the local heating by the electron beam ${ }^{34}$. Similar redshifts were also observed from other TMDCs, such as $\mathrm{MoS}_{2}$ and $\mathrm{WS}_{2}$, studied in this work. The patchy emission pattern within the sandwiched region in Figure $2 \mathrm{c}$ is due to poor interface contacts developed during the dry transfer. The $\mathrm{CL}$ intensity was proportional to the beam current and remained linear within the available beam current (Supporting Information Figure S2e).

a

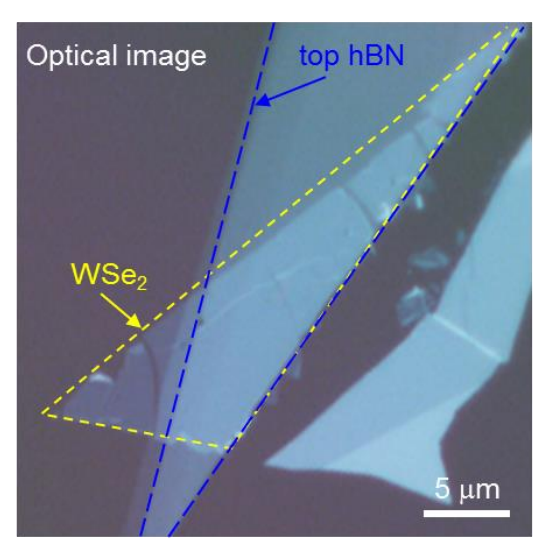

C

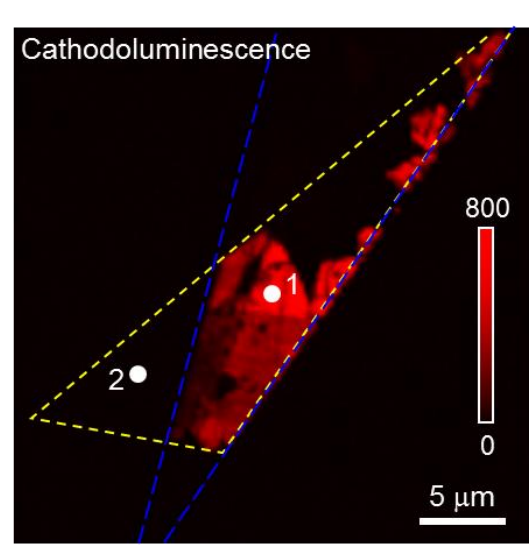

$\mathrm{b}$
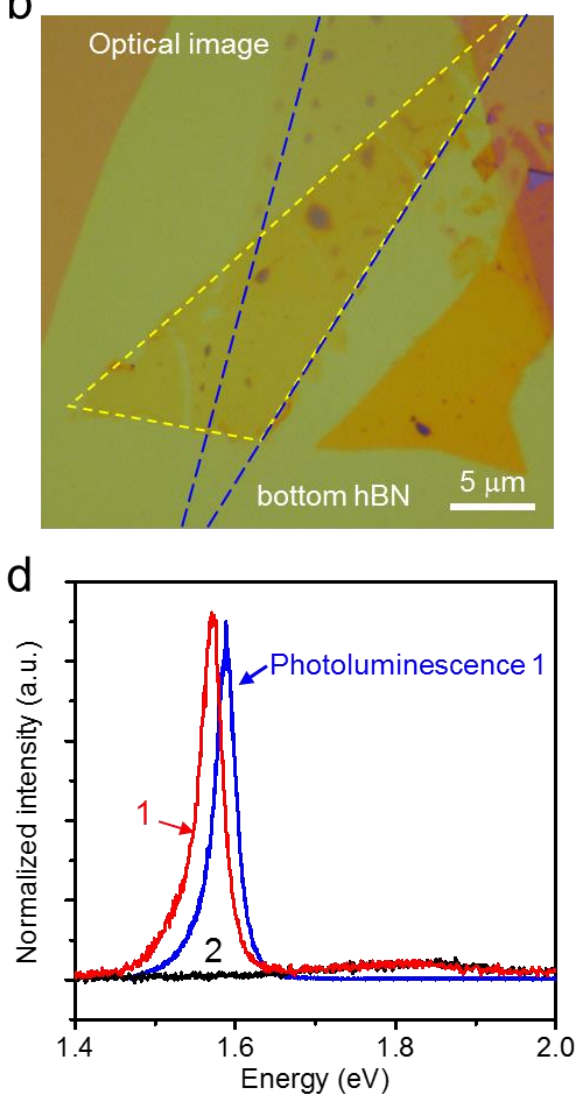

Figure 2. Optical characterization of a heterostructure $\mathbf{h B N} / \mathrm{WSe} / \mathrm{hBN}$. Optical microscope images of a heterostructure $\mathrm{hBN} / \mathrm{WSe} \mathrm{L}_{2} / \mathrm{hBN}$ (a) before and (b) after the final dry transfer onto a bottom hBN layer. While Top hBN flake (blue) and monolayer $\mathrm{WSe}_{2}$ (yellow) can be identified as small flakes, the edge of larger bottom hBN flake is not seen in the images. (c) Monochromatic $\mathrm{CL}$ map of the heterostructure at $\mathrm{WSe}_{2}$ emission energy, $1.66 \mathrm{eV}$ (colour coded in red). (d) CL spectra generated by electron beam impact on $\mathrm{WSe}_{2}$ at position 1 and 2 in (c) are shown together with a PL spectrum acquired at position 1 in (c).

\section{Effect of hBN thickness on CL enhancement}

In electroluminescence or PL configuration, hBN layers in such heterostructures would only work as tunneling barriers for electrically or optically injected charge carriers. In CL configuration, they should also work as a source for extra e-h pairs due to the broadband 
pumping capability of swift electrons. Part of them can diffuse into and trapped in the sandwiched monolayer TMDCs before they recombine in hBN layers. To verify this aspect of $\mathrm{hBN}$ layers, we prepared two types of heterostructures where either the top or bottom hBN layer is manipulated to have a selection of thickness and studied the effect of barrier thickness on the CL intensity.

To see the effect of top $\mathrm{hBN}$ layer, a heterostructure $\mathrm{hBN} / \mathrm{WSe} 2 / \mathrm{hBN}$ was fabricated from a bottom hBN layer with a uniform thickness of $165.3 \mathrm{~nm}$ and a top hBN layer with three regions of different thickness from 3.5, 11.8, and $23.0 \mathrm{~nm}$. The CL mapping for $\mathrm{WSe}_{2}$ emission shows a clear contrast in intensity between the three regions (see Supporting Information Figure S3a-c). Figure 3a shows CL spectra from the three regions where each spectrum is an average of multiple spectra acquired from 20 positions within each region to remove the intensity inhomogeneity. The peak CL intensity increases with the top $\mathrm{hBN}$ thickness in a nearly linear fashion (Figure $3 b$ ).

To see the effect of bottom hBN layer, another heterostructure $\mathrm{hBN} / \mathrm{WSe} 2 / \mathrm{hBN}$ which was prepared from a top hBN layer with a uniform thickness of $20.0 \mathrm{~nm}$ and a bottom hBN layer with four regions of thickness from 12.1, 21.6, 36.7, $48.3 \mathrm{~nm}$. The CL mapping shows a similar dependence of increasing CLintensity with bottom hBN thickness (Figure 3c-d and Supporting Information Figure S3d-f). The slight variations in CL peak positions between regions in both samples might be arising from local strain and/or heterostructure inhomogeneity. The dependence of CL intensity on the top and bottom hBN thickness was also confirmed in another set of samples. This dependence confirms our model in Figure 1 that majority of e-h pairs which recombine in TMDC monolayers originate from the adjacent hBN layers. The diffusion lengths of electrons and holes in $\mathrm{hBN}$ are known to be in the $\mu \mathrm{m}$ range, ${ }^{35}$ so large amount of e$\mathrm{h}$ pairs created in both top and bottom $\mathrm{hBN}$ layers in the heterostructure can diffuse into the TMDC monolayer layer before recombination. As hBN layers with larger thickness would support larger interaction volume for e-h pair creation, the amount of e-h pairs that eventually trapped in TMDC monolayer should be proportional to the hBN layer thickness. It is noteworthy that the bottom $\mathrm{hBN}$ layer cannot be replaced by the amorphous $\mathrm{SiO}_{2}$ substrate, as the latter does not provide an atomically flat surface as well as perfect contact. In order to roughly estimate the enhancement factor in our experiments, we compare the intensity ratio of 
the sandwiched $\mathrm{hBN} / \mathrm{WSe} / \mathrm{hBN}$ to that of the $\mathrm{WSe} 2 / \mathrm{hBN}$ (i.e., with only bottom $\mathrm{hBN}$ ), which is more than 500 times (see Figure 3e).
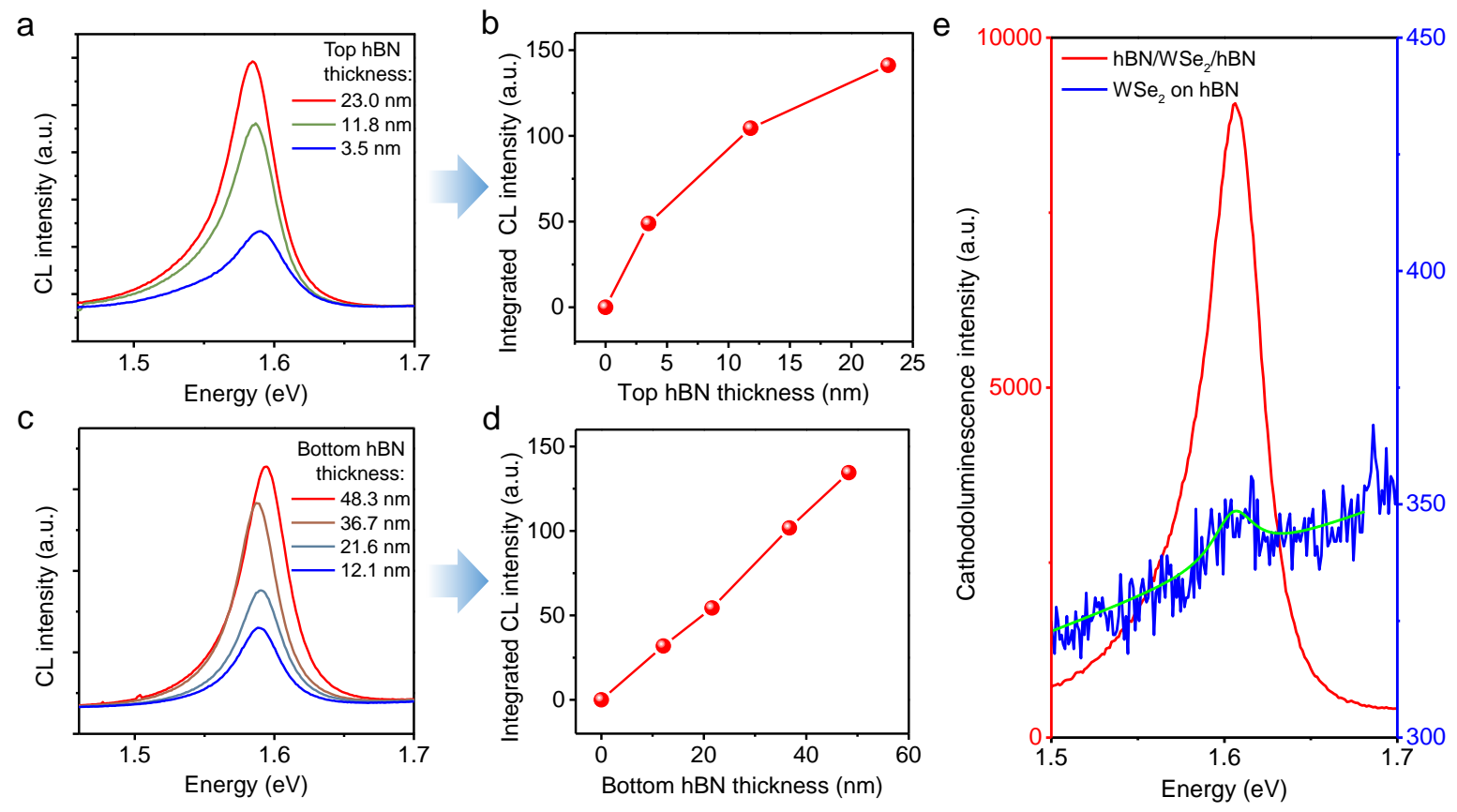

Figure 3. Effects of hBN layers on CL intensity. CL spectra acquired from regions with different (a) top and (c) bottom hBN thickness. The CL integrated intensity is shown as a function of (b) top and (d) bottom hBN thickness. (e) Comparison of CL intensity of sandwiched $\mathrm{WSe}_{2}$ (with $19.8 \mathrm{~nm}$ top and $123.9 \mathrm{~nm}$ bottom $\mathrm{hBN}$ layers, red line) and $\mathrm{WSe}_{2}$ with only bottom $\mathrm{hBN}$ layer (blue line). Green line is the Lorenz fitting of the blue line. The enhancement factor is estimated to be more than 500 .

\section{Nanoscale imaging of strain-induced band gap variation}

To demonstrate the full potential of CL spectroscopy as a nanoscale optical characterization tool for monolayer TMDCs, we created a heterostructure $\mathrm{hBN} / \mathrm{WSe} / \mathrm{hBN}$ possessing nanoscale variation of strain by placing the heterostructure on a pre-patterned $\mathrm{Si}$ substrate with holes of 1-2 $\mu$ m diameter (see inset of Figure 4a and Supporting Information Figure S4). Part of the heterostructure sitting above the holes are suspended and develops spatially-varying strain which induces band gap shrinkage of the monolayer $\mathrm{WSe}_{2}$ accordingly. This strain-induced band-gap engineering is well established both theoretically ${ }^{36}$ and experimentally ${ }^{37-39}$. Interestingly, the CL intensity from the suspended part of the heterostructure is much higher than the rest of the heterostructure (Figure 4a). This is attributed to the funnel effect ${ }^{40}$ where the e-h pairs in the monolayer drift toward the strained part due to 
the lowered band gap and recombine. To enhance the spectral resolution for resolving the strain-induced peak shifts, the sample was cooled down to $10 \mathrm{~K}$ where the exciton and trion peaks well separated. Position-dependent spectra at $10 \mathrm{~K}$ (Figure $4 \mathrm{~b}$ and $4 \mathrm{c}$ ) clearly shows that the excitonic peak redshift with a maximum shift of $11.2 \mathrm{meV}$ from the edge to the center of the hole due to strain.
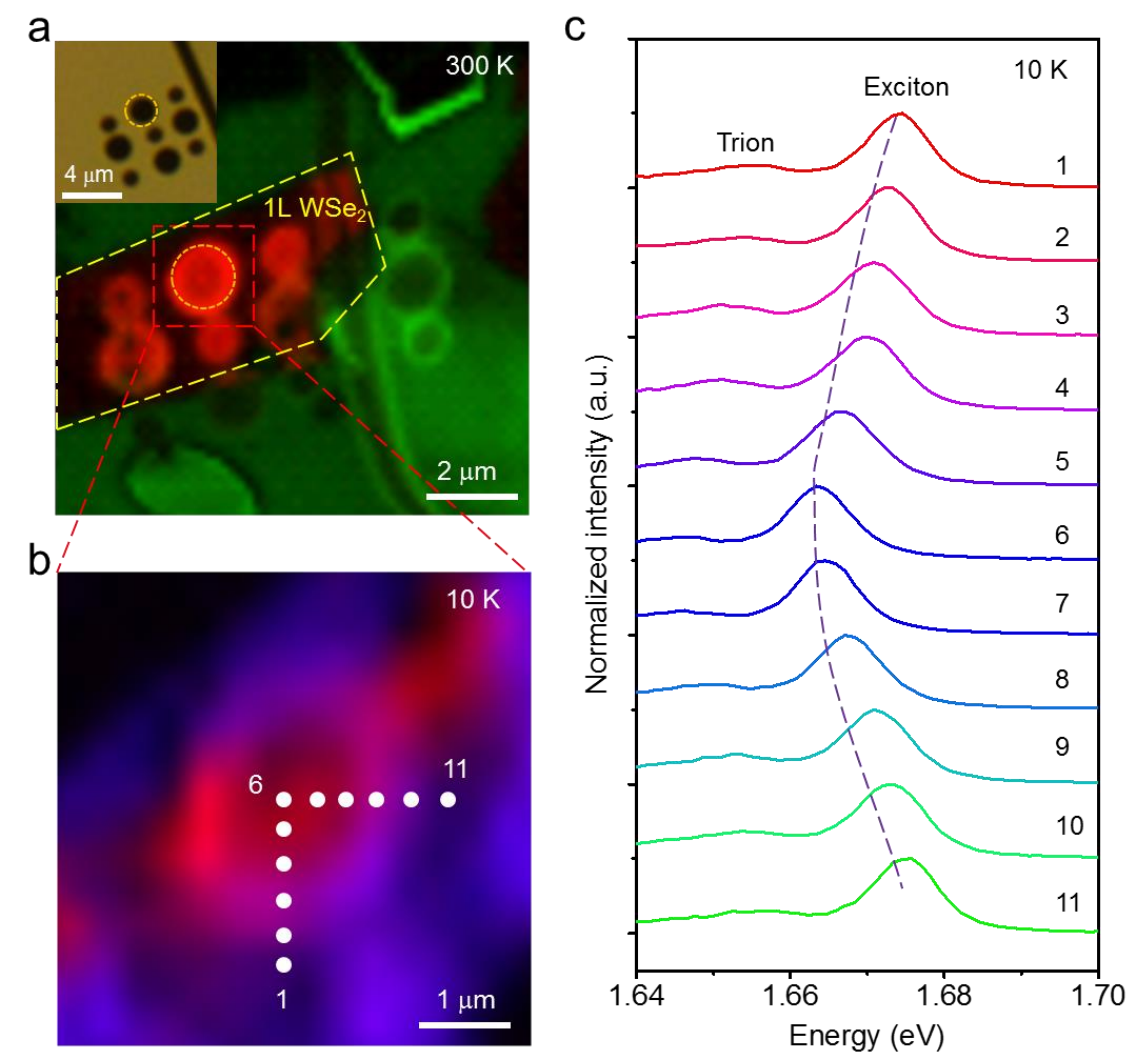

Figure 4. Imaging of the strain-induced band gap variation. (a) CL image of an $\mathrm{hBN} / \mathrm{WSe}_{2} / \mathrm{hBN}$ heterostructure on a patterned $\mathrm{Si}$ substrate at room temperature. The pseudocolors correspond to the luminescence in 307-331 nm (green) and 733-788 nm (red). Inset: Scanning electron microscopy image of the patterned silicon substrate without the heterostructure.

(b) CL mapping acquired for the hole highlighted in (a) with higher magnification at $10 \mathrm{~K}$. The pseudo-colors correspond to the luminescence in 707-716 nm (blue) and 716-726 nm (red). (c) Position-dependent spectra taken from a selection of data points in (b).

In addition to the strain induced by suspension, local strain can be introduced in the heterostructures during the transfer process. Such inhomogeneous local strain in the heterostructures is also detectable by CL spectroscopy. Indeed two energy domains were observed from the $\mathrm{hBN} / \mathrm{WSe} / \mathrm{hBN}$ (indicated by purple and green colors in Supporting Information Figure S5a) sample in terms of the exciton peak position at $77 \mathrm{~K}$. From the CL 
spectra from selected points (Supporting Information Figure S5b), two emission peaks can be resolved at both point $\mathrm{A}$ and $\mathrm{B}$. The two peaks correspond to the emissions of neutral excitons and trions (charged excitons) ${ }^{41}$. However, the peak positions of excitons and trions at point A are 1.640 and $1.614 \mathrm{eV}$, respectively, while 1.657 and $1.623 \mathrm{eV}$ at point $\mathrm{B}$. The peak position difference between point $\mathrm{A}$ and $\mathrm{B}$ in the heterostructure may be caused by strain, which is possibly generated during the transfer process (e.g., bubbles).

Temperature dependent CL spectra of the point B were plotted (Supporting Information Figure S5c). The peak positions of both excitons and trions are fitted (Supporting Information Figure S5d) according to the semi-empirical semiconductor band gap equation ${ }^{33,42}$ of $E_{g}(T)=$ $E_{g}(0)-S \hbar \omega\left[\operatorname{coth}\left(\frac{\hbar \omega}{2 k T}\right)-1\right]$, where $E_{g}(0)$ is the excitonic energy at $0 \mathrm{~K}, S$ is a dimensionless coupling constant and $\hbar \omega$ is an average phonon energy. From the fitting curves, we extract $E_{g}(0)$ of the exciton and trion of the monolayer $\mathrm{WSe}_{2}$ to be 1.665 and $1.634 \mathrm{eV}$, respectively. So, the binding energy of the trion is calculated to be $\sim 30.8 \mathrm{meV}$ which is consistent with the previous report $(30 \mathrm{meV})^{32}$.

\section{CL enhancement in heterostructures with $\mathrm{MoS}_{2}$ and $\mathrm{WS}_{2}$}

In addition to $\mathrm{WSe}_{2}$, we also performed CL experiments to monolayer $\mathrm{WS}_{2}$ and $\mathrm{MoS}_{2}$ in a van der Waals heterostructure (sandwiched by two hBN layers). The emission peak position from the $\mathrm{WS}_{2}$ heterostructure locates at $1.933 \mathrm{eV}$ (Figure 5a) and that from the $\mathrm{MoS}_{2}$ in heterostructure at $1.831 \mathrm{eV}$ (Figure 5b). The emission peak positions of both two heterostructures redshift with respect to their PL peak positions, similar to the case of $\mathrm{hBN} / \mathrm{WSe}_{2} / \mathrm{hBN}$. The CL mapping is inhomogeneous at the $\mathrm{MoS}_{2}$ sample, which is most likely due to a poor interface contact. Therefore, we have attested that sandwiching a monolayer TMDC into two hBN layers is a universal approach to study its CL emission.

In summary, for the first time we have obtained evident CL emissions from monolayer TMDCs, including $\mathrm{WSe}_{2}, \mathrm{MoS}_{2}$ and $\mathrm{WS}_{2}$, via a van der Waals heterostructure configuration. In the $\mathrm{hBN} / \mathrm{TMDC} / \mathrm{hBN}$ heterostructure, electron beam induced e-h pairs can transfer to and be trapped in the middle TMDC layer, leading to increased recombination probability within the TMDC layer. Moreover, we demonstrate that CL spectroscopy can be applied to study the 
strain-induced excitonic peak shift in monolayer TMDCs. Because of its high spatial resolution and high beam energy, our demonstration makes CL spectroscopy a powerful technique to the study of 2D materials in various forms such as alloy, heterostructures or defects. The 2D monolayer-based heterostructure may promise potential applications in single-photon emitters, surface-conduction electron-emitter and field emission display technologies.
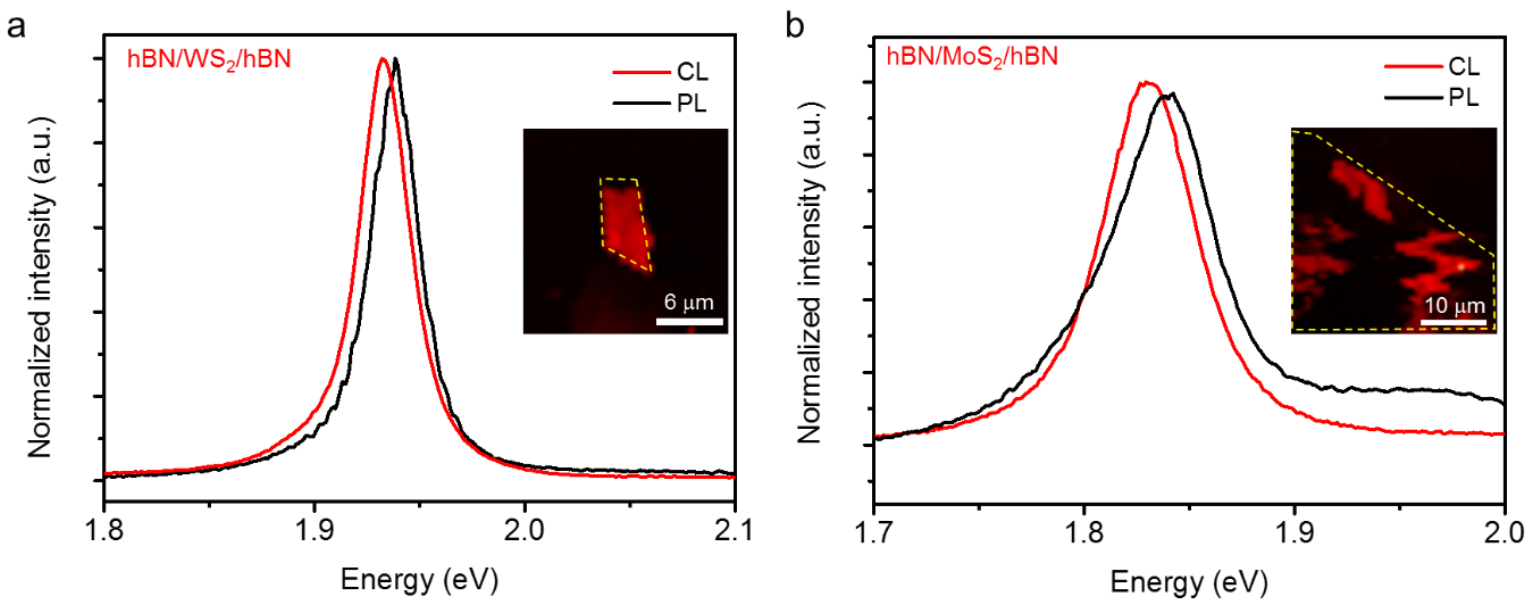

Figure 5. Cathodoluminescence of monolayer $\mathbf{W S}_{\mathbf{2}}$ and $\mathbf{M o S}_{2}$. (a) CL and PL spectra of the monolayer $\mathrm{WS}_{2}$ in the top-hBN $(7.5 \mathrm{~nm}) / \mathrm{WS}_{2} /$ bottom-hBN $(299.4 \mathrm{~nm})$. (b) CL and PL spectra of the monolayer $\mathrm{MoS}_{2}$ in the top-hBN $(13.5 \mathrm{~nm}) / \mathrm{MoS}_{2} / \mathrm{bottom}-\mathrm{hBN}(168.6 \mathrm{~nm})$. Insets are the corresponding CL intensity mappings.

\section{Methods}

\section{Heterostructure preparation}

Heterostructures of hBN/TMDC/hBN were prepared using a dry transfer technique ${ }^{1,32} . \mathrm{hBN}$ and monolayer TMDC flakes were mechanically exfoliated from bulk hBN and TMDC single crystals synthesized by chemical vapor transport method with Scotch tapes and deposited on $300 \mathrm{~nm}$-thick $\mathrm{SiO}_{2}$ on $\mathrm{Si}\left(\mathrm{SiO}_{2} / \mathrm{Si}\right)$ substrates. Mono- and few-layer flakes were identified by optical contrast (Nikon optical microscope), Raman spectroscopy and atomic force microscopy (AFM) (see Supporting Information Figure S2). As adhesion layer, polyvinyl alcohol (PVA) was used as it is water-soluble with a moderate adhesion. A PVA solution (9\% weight in water) was spin coated on a polydimethylsiloxane (PDMS) film ( $0.5 \mathrm{~mm}$ thick). After baking at $90{ }^{\circ} \mathrm{C}$ on a hot plate, the PDMS/PVA film was attached on a glass slide and the whole stack was mounted on a micromanipulator. Under an optical microscope, the 
PDMS/PVA stack was aligned to an $\mathrm{hBN}$ flake on $\mathrm{a} \mathrm{SiO}_{2} / \mathrm{Si}$ substrate and brought into contact with the flake underneath. The flake can be easily picked up due to its stronger adhesion to PVA than $\mathrm{SiO}_{2}$. The procedure was repeated to pick up a monolayer TMDC flake. Then, the hBN/TMDC on the stack was aligned and brought into contact to another $\mathrm{hBN}$ flake on a $\mathrm{Si} / \mathrm{SiO}_{2}$ substrate. The PDMS/PVA film was released from the heterostructure on $\mathrm{SiO}_{2} / \mathrm{Si}$ by slowly peeling the PDMS film from the PVA film at $70{ }^{\circ} \mathrm{C}$ leaving the PVA film on the $\mathrm{Si} / \mathrm{SiO}_{2}$. The PVA film was washed off by dipping in water for half an hour. Fewer bubbles and better contacts were created with the latter approach since the heterostructure was not stretched during the removal of the PDMS film. For the patterning of the $\mathrm{Si}$ substrate in Figure 4, a focused ion beam (FEI Helios NanoLab) was used to mill holes with 1-2 $\mu \mathrm{m}$ diameter and $1 \mu \mathrm{m}$ depth.

\section{AFM and optical characterization}

The thickness of hBN flakes was measured using a Cypher ES scanning probe microscopy in AC tapping mode. A Witec alpha300 R confocal Raman spectrum was used to collect Raman and photolulminescence spectra where a pump laser at $532 \mathrm{~nm}$ at power of $0.2 \mathrm{~mW}$ was used to illuminate the samples through a $\times 100$ objective.

\section{CL measurement}

CL measurements were performed in a scanning electron microscope equipped with a CL detection system, Attolight Allalin 4027 Chronos. A focused electron beam (electron energy $5 \mathrm{keV}$; beam current 36 nA; dewell time 10-50 ms) scanned the samples while recording the light emission spectrum synchronously to produce hyperspectral images. The emitted light was collected by an achromatic reflective objective with a high numerical aperture (NA 0.72) and sent to a UV-VIS spectrometer (Horiba iHR320) equipped with a thermoelectrically cooled silicon CCD array (Andor Newton). For low temperature measurements, samples were cooled down with an open-cycle cryostat by continuously flowing either liquid nitrogen or liquid helium.

\section{ASSOCIATED CONTENT}

Supporting Information Available: Further information on sample transfer, more AFM and optical images, and CL spectra. This material is available free of charge via the Internet at http://pubs.acs.org.

\section{AUTHOR INFORMATION}




\section{Corresponding Authors.}

*Email: NZheludev@ntu.edu.sg (N. Z)

*Email: fanhj@ntu.edu.sg (H.J. F);

\section{Author contributions}

SZ conceived the idea of using vdW heterostructures and prepared samples. JKS and SZ conducted CL measurements. FL provided the hBN and TMDCs crystals and conducted Raman and PL measurements. SZ and JKS prepared the manuscript. All authors contributed to the analysis and interpretation of results and writing of the paper. HJF supervised the project. "These authors contributed equally.

\section{Notes}

The authors declare no competing financial interest.

\section{ACKNOWLEDGEMENTS}

This work was supported by Singapore Ministry of Education Academic Research Fund Tier 3 (Grant No. MOE2011-T3-1-005), the Singapore National Research Foundation under NRF RF Award No. NRF-RF2013-08, the start-up funding from Nanyang Technological University (M4081137.070), and Nanyang Technological University Strategic Research Facilities Initiative.

\section{References}

1. Castellanos-Gomez, A.; Buscema, M.; Molenaar, R.; Singh, V.; Janssen, L.; van der Zant, H. S. J.; Steele, G. A. 2D Materials 2014, 1, (1), 011002.

2. Chhowalla, M.; Shin, H. S.; Eda, G.; Li, L. J.; Loh, K. P.; Zhang, H. Nat Chem 2013, 5, (4), 263-275.

3. Novoselov, K. S.; Geim, A. K.; Morozov, S. V.; Jiang, D.; Zhang, Y.; Dubonos, S. V.; Grigorieva, I. V.; Firsov, A. A. Science 2004, 306, (5696), 666-669.

4. Dean, C. R.; Young, A. F.; Meric, I.; Lee, C.; Wang, L.; Sorgenfrei, S.; Watanabe, K.; Taniguchi, T.; Kim, P.; Shepard, K. Nat Nanotechnol 2010, 5, (10), 722-726.

5. Zeng, H. L.; Dai, J. F.; Yao, W.; Xiao, D.; Cui, X. D. Nat Nanotechnol 2012, 7, (8), 490-493.

6. Shan, W. Y.; Lu, H. Z.; Xiao, D. Phys Rev B 2013, 88, (12), 125301.

7. Britnell, L.; Gorbachev, R.; Geim, A.; Ponomarenko, L.; Mishchenko, A.; Greenaway, M.; Fromhold, T.; Novoselov, K.; Eaves, L. Nat Commun 2013, 4, 1794.

8. Nguyen, L.-N.; Lan, Y.-W.; Chen, J.-H.; Chang, T.-R.; Zhong, Y.-L.; Jeng, H.-T.; Li, L.-J.; Chen, C.-D. Nano Lett 2014, 14, (5), 2381-2386.

9. Campbell, P. M.; Tarasov, A.; Joiner, C. A.; Ready, W. J.; Vogel, E. M. Acs Nano 2015, 9, (5), 5000-5008.

10. Withers, F.; Del Pozo-Zamudio, O.; Mishchenko, A.; Rooney, A. P.; Gholinia, A.; Watanabe, K.; Taniguchi, T.; Haigh, S. J.; Geim, A. K.; Tartakovskii, A. I.; Novoselov, K. S. Nat Mater 2015, 14, (3), 301-306.

11. Helveg, S.; Lauritsen, J. V.; Laegsgaard, E.; Stensgaard, I.; Norskov, J. K.; Clausen, B. S.; Topsoe, H.; Besenbacher, F. Phys Rev Lett 2000, 84, (5), 951-954. 
12. Van Der Zande, A. M.; Huang, P. Y.; Chenet, D. A.; Berkelbach, T. C.; You, Y.; Lee, G.-H.; Heinz, T. F.; Reichman, D. R.; Muller, D. A.; Hone, J. C. Nat Mater 2013, 12, (6), 554-561.

13. Bao, W.; Melli, M.; Caselli, N.; Riboli, F.; Wiersma, D.; Staffaroni, M.; Choo, H.; Ogletree, D.; Aloni, S.; Bokor, J. Science 2012, 338, (6112), 1317-1321.

14. Boggs, S.; Krinsley, D., Application of cathodoluminescence imaging to the study of sedimentary rocks. Cambridge University Press: 2006, p7.

15. Chin, A. K.; Zipfel, C. L.; Mahajan, S.; Ermanis, F.; Digiuseppe, M. A. Appl Phys Lett 1982, 41, (6), 555557.

16. Griffiths, J. T.; Zhang, S.; Rouet-Leduc, B.; Fu, W. Y.; Bao, A.; Zhu, D.; Wallis, D. J.; Howkins, A.; Boyd, I.; Stowe, D.; Kappers, M. J.; Humphreys, C. J.; Oliver, R. A. Nano Lett 2015, 15, (11), 7639-43.

17. Zhao, M.; Bosman, M.; Danesh, M.; Zeng, M.; Song, P.; Darma, Y.; Rusydi, A.; Lin, H.; Qiu, C. W.; Loh, K. P. Nano Lett 2015, 15, (12), 8331-8335.

18. Ruf, T.; Cardona, M.; Sternschulte, H.; Wahl, S.; Thonke, K.; Sauer, R.; Pavone, P.; Anthony, T. R. Solid State Commun 1998, 105, (5), 311-316.

19. Watanabe, K.; Taniguchi, T.; Kanda, H. Nat Mater 2004, 3, (6), 404-409.

20. Arnaud, B.; Lebegue, S.; Rabiller, P.; Alouani, M. Phys Rev Lett 2006, 96, (2), 026402.

21. Bashevoy, M.; Jonsson, F.; MacDonald, K.; Chen, Y.; Zheludev, N. Opt Express 2007, 15, (18), 11313-11320.

22. Tikhomirov, V.; Adamo, G.; Nikolaenko, A.; Rodriguez, V.; Gredin, P.; Mortier, M.; Zheludev, N.; Moshchalkov, V. Opt Express 2010, 18, (9), 8836-8846.

23. Denisyuk, A.; Adamo, G.; MacDonald, K.; Edgar, J.; Arnold, M.; Myroshnychenko, V.; Ford, M.; García de Abajo, F. J.; Zheludev, N. Nano Lett 2010, 10, (9), 3250-3252.

24. Xu, J.; Chen, L.; Yu, L.; Liang, H.; Zhang, B.; Lau, K. M. Journal of Electronic Materials 2007, 36, (9), 1144-1148.

25. Priesol, J.; Satka, A.; Uherek, F.; Donoval, D.; Shields, P.; Allsopp, D. W. E. Applied Surface Science 2013, $269,155-160$.

26. Atre, A. C.; Brenny, B. J.; Coenen, T.; Garcia-Etxarri, A.; Polman, A.; Dionne, J. A. Nat Nanotechnol 2015, $10,(5), 429-436$.

27. Adamo, G.; MacDonald, K. F.; Fu, Y.; Wang, C.; Tsai, D.; de Abajo, F. G.; Zheludev, N. Phys Rev Lett 2009, 103, (11), 113901.

28. Li, G.; Clarke, B. P.; So, J. K.; MacDonald, K. F.; Zheludev, N. I. Nat Commun 2016, 7, 13705.

29. Bourrellier, R.; Amato, M.; Tizei, L. H. G.; Giorgetti, C.; Gloter, A.; Heggie, M. I.; March, K.; Stephan, O.; Reining, L.; Kociak, M.; Zobelli, A. Acs Photonics 2014, 1, (9), 857-862.

30. Watanabe, K.; Taniguchi, T.; Niiyama, T.; Miya, K.; Taniguchi, M. Nat Photonics 2009, 3, (10), $591-594$.

31. Schue, L.; Berini, B.; Betz, A. C.; Placais, B.; Ducastelle, F.; Barjon, J.; Loiseau, A. Nanoscale 2016, 8, (13), 6986-6993.

32. Pizzocchero, F.; Gammelgaard, L.; Jessen, B. S.; Caridad, J. M.; Wang, L.; Hone, J.; Boggild, P.; Booth, T. J. Nat Commun 2016, 7, 11894.

33. O’Donnell, K.; Chen, X. Appl Phys Lett 1991, 58, (25), 2924-2926.

34. Rodriguez-Viejo, J.; Jensen, K.; Mattoussi, H.; Michel, J.; Dabbousi, B.; Bawendi, M. Appl Phys Lett 1997, 70, (16), 2132-2134.

35. Maity, A.; Doan, T. C.; Li, J.; Lin, J. Y.; Jiang, H. X. Appl Phys Lett 2016, 109, (7), 072101.

36. Amin, B.; Kaloni, T. P.; Schwingenschlogl, U. Rsc Adv 2014, 4, (65), 34561-34565.

37. Castellanos-Gomez, A.; Roldán, R.; Cappelluti, E.; Buscema, M.; Guinea, F.; van der Zant, H. S.; Steele, G. A. Nano Lett 2013, 13, (11), 5361-5366. 
38. Conley, H. J.; Wang, B.; Ziegler, J. I.; Haglund, R. F., Jr.; Pantelides, S. T.; Bolotin, K. I. Nano Lett 2013, 13, (8), 3626-3630.

39. Desai, S. B.; Seol, G.; Kang, J. S.; Fang, H.; Battaglia, C.; Kapadia, R.; Ager, J. W.; Guo, J.; Javey, A. Nano Lett 2014, 14, (8), 4592-4597.

40. Feng, J.; Qian, X.; Huang, C.-W.; Li, J. Nat Photonics 2012, 6, (12), 866-872.

41. Wang, G.; Bouet, L.; Lagarde, D.; Vidal, M.; Balocchi, A.; Amand, T.; Marie, X.; Urbaszek, B. Phys Rev B 2014, 90, (7), 075413.

42. Ross, J. S.; Wu, S.; Yu, H.; Ghimire, N. J.; Jones, A. M.; Aivazian, G.; Yan, J.; Mandrus, D. G.; Xiao, D.; Yao, W.; Xu, X. Nat Commun 2013, 4, 1474. 


\section{For Table of Contents Only}
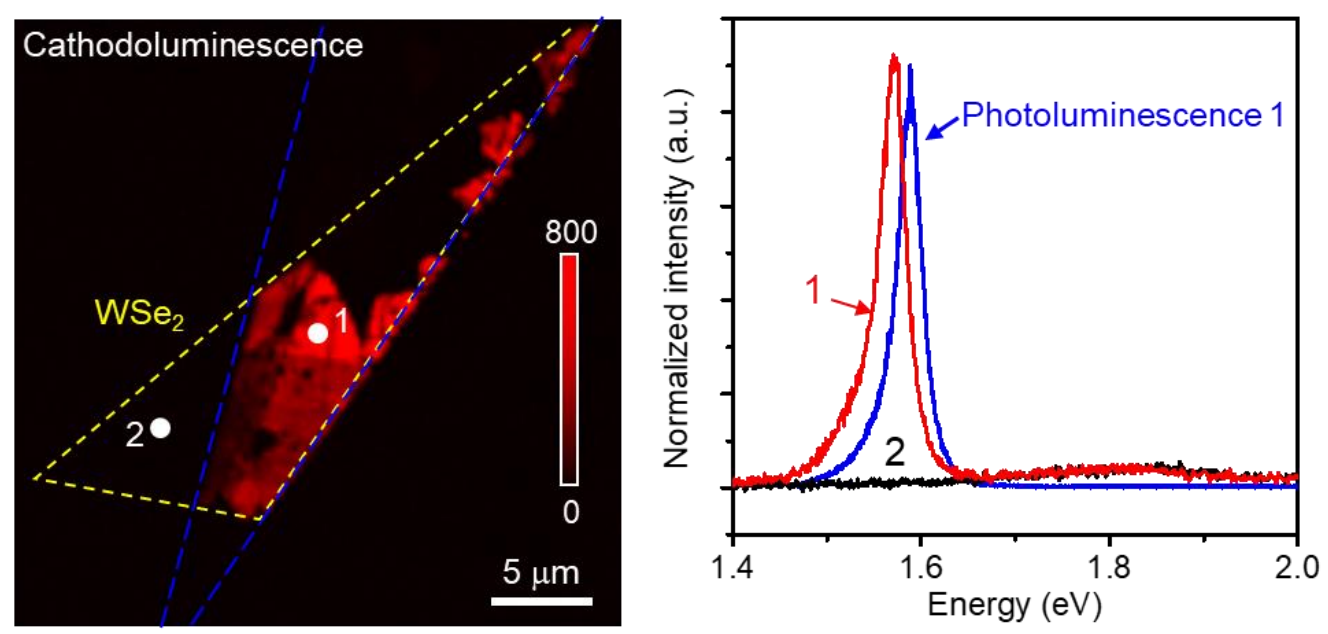